\title{
Prise au piège de la folie ? Le pouvoir étatique et le crime organisé transnational dans l'Suvre de Susan Strange
}

Richard Friman

\section{(2) OpenEdition Journals}

Édition électronique

URL : http://journals.openedition.org/conflits/564

DOI : $10.4000 /$ conflits.564

ISSN : $1777-5345$

Éditeur :

CCLS - Centre d'études sur les conflits lilberté et sécurité, L'Harmattan

Édition imprimée

Date de publication : 1 juin 2001

ISBN : 2-7475-1117-0

ISSN : $1157-996 \mathrm{X}$

Référence électronique

Richard Friman, «Prise au piège de la folie ? Le pouvoir étatique et le crime organisé transnational dans I'Suvre de Susan Strange », Cultures \& Conflits [En ligne], 42 | été 2001, mis en ligne le 13 mars 2006, consulté le 30 mars 2021. URL : http://journals.openedition.org/conflits/564 ; DOI : https:// doi.org/10.4000/conflits.564

Ce document a été généré automatiquement le 30 mars 2021.

Creative Commons License 


\title{
Prise au piège de la folie? Le pouvoir étatique et le crime organisé transnational dans l'Suvre de Susan Strange
}

\author{
Richard Friman
}

Susan Strange a laissé une œuvre impressionnante sur les relations internationales (Lawton, Rosenau, Verdun 2000$)^{1}$. Elle a régulièrement remis en question la tendance de cette discipline à privilégier l'Etat et à négliger le rôle des marchés et des acteurs transnationaux. Les acteurs étatiques et non-étatiques se disputent selon elle le pouvoir, un thème central de son œuvre étant les négociations entre ces acteurs dans le contexte des relations transnationales. Dans deux de ses derniers ouvrages majeurs, The Retreat of the State: The Diffusion of Power in the World Economy (1996) et Mad Money (1998), elle a élargi son approche aux défis posés au pouvoir étatique par le crime organisé transnational. Cette démarche constituait un risque professionnel à de nombreux égards. Le crime transnational se situe à la marge de l'étude des relations internationales et constitue un domaine souvent considéré comme trop journalistique, handicapé par l'absence de données fiables, et, dans certains cas, présentant trop de dangers pour que le chercheur continue ses investigations. Les travaux de Strange dans ce contexte ont contribué à légitimer la recherche universitaire sur ce thème. Sa trop grande confiance dans la discipline même qu'elle tentait de remettre en cause limite néanmoins sa perspicacité. Les problèmes en jeu sont pour le moins de deux ordres et tournent autour des questions de théorie et de menace. Dans le premier domaine, Strange reproche à la discipline des relations internationales l'adoption d'une approche centrée sur l'Etat faussant quelque peu ses concepts et ses hypothèses. Elle n'en tombe pas moins dans des pièges similaires en se fondant sur des éléments de cette approche pour expliquer la nature et l'influence des acteurs non-étatiques. Sur le deuxième point, la menace, elle critique cette même approche pour son absence de prise en compte de l'érosion de l'autorité étatique face au crime transnational. Or elle accepte largement, en les posant comme donnés, les arguments des acteurs étatiques 
concernant la nature et l'étendue de cette érosion. Négligeant d'étudier le degré de construction sociale des menaces du crime et la variété d'acteurs étatiques ou non impliqués dans ce processus, Strange ne remplit pas son objectif de proposer une recherche critique des menaces posées par le crime organisé transnational. La première partie de ce compte rendu résumera brièvement les arguments principaux de Strange tels qu'elle les a présentés dans The Retreat of the State et Mad Money. Les suivantes traiteront de son approche des questions de théorie et de menace. Retrait de l'Etat et folie financière L'idée que le pouvoir se disperse de plus en plus dans l'économie mondiale constitue un thème central des deux ouvrages et le sous-titre du premier. Strange observe une évolution selon laquelle le pouvoir se propage des acteurs étatiques aux non-étatiques et, plus largement, aux marchés mondiaux. Ce processus de « diffusion » est complexe, façonné par l'interaction du progrès technique, des marchés financiers et des décisions ou de l'absence de décision des acteurs étatiques et non étatiques (1996, pp. 42-43, pp. 185-186; 1998, pp. 9-10). Les arguments de Strange sur le retrait de l'Etat et la folie des marchés financiers qui en résulte poursuivent ceux de son ouvrage précédent sur la nature du pouvoir et de la politique (Strange 1988). Nous allons brièvement discuter ces concepts dans cette partie avant de nous pencher sur ses arguments relatifs aux défis posés par le crime organisé transnational. Strange a cherché à élargir les paramètres de l'étude des relations internationales lorsqu'elle a conceptualisé le pouvoir et la politique. Dans The Retreat of the State, elle se fonde sur ces thèmes en observant que l'importance du pouvoir repose moins sur les potentiels ou les ressources relatives des Etats cherchant à s'influencer les uns les autres, que sur la capacité des acteurs étatiques et non-étatiques à influencer des structures plus larges de « relations de pouvoir » (1996, p. 23). Strange définit le pouvoir structurel en termes d'autorité ou de "pouvoir sur " des résultats dans un cadre mondial de relations de sécurité, de finances, de production et de savoir. Le pouvoir structurel implique la capacité d'«affecter des résultats» de sorte que « les préférences [d'une personne ou d'un groupe] prennent le pas sur celles des autres » $(1996$, p.17). Strange ouvre la voie à son argument plus vaste sur la diffusion du pouvoir en affirmant que les acteurs étatiques et non-étatiques sont en mesure d'exercer un pouvoir structurel. Ces acteurs peuvent chercher consciemment à affecter les résultats, ou le font « inconsciemment ", dans le cas d'acteurs étatiques et non-étatiques puissants, par la simple vertu de leur présence (1996, pp. 17-19, pp. 25-26). Les acteurs puissants modèlent la politique. Pour Strange, les acteurs tant étatiques que non-étatiques sont engagés dans un comportement politique sur lequel ils impriment leur marque. Elle définit la politique de façon large comme "toutes les activités par lesquelles des personnes sont persuadées ou forcées de collaborer à la réalisation de buts désignés et désirés par un tiers » (1996, p. 53). Elle affirme que les buts en question, pour les acteurs tant étatiques que non-étatiques, impliquent quatre " valeurs » majeures, la fourniture de sécurité, de richesse, de justice et de liberté et/ou d'autonomie (1996, p. 35). Les activités politiques sont celles qui déterminent la «combinaison des valeurs dans le système dans son ensemble et leur répartition parmi les groupes sociaux » (1996, p. 34). En définissant la politique comme l'exercice d'une « autorité sur l'attribution de valeur » par les acteurs étatiques et non-étatiques, Strange se trouve en mesure d'étudier la question de savoir qui contrôle vraiment cette autorité et d'affirmer l'existence d'un processus de diffusion du pouvoir (1996, p.35). La thèse la plus importante de The Retreat of the State et de Mad Money est que les Etats perdent le contrôle de l'autorité en matière d'attribution de valeur au profit d'autres Etats, d'acteurs non-étatiques et du marché 
(par exemple 1996, pp. 29-30, pp. 42-43 ; 1998, pp. 43-44, pp. 60-74, p. 78, p. 98, p. 180, p. 190). Cette diffusion varie selon les domaines de pouvoir structurel et la nature (notamment la taille, la force et la centralisation) de l'Etat (1996, p. ix, xii, pp. 13-14, p. 25, p. 92). Pour saisir ce phénomène, Strange propose une ébauche de continuum à partir du degré du défi posé à l'autorité étatique par les acteurs non-étatiques. Ce défi peut être «directement le fait du pouvoir relationnel, ou indirectement celui du pouvoir structurel » (1996, p. 91). Elle soutient que le crime organisé transnational se place à une extrémité de ce continuum comme « une autorité non-étatique qui conteste et défie, ou menace de supplanter l'Etat» $(1996$, p. $92 ; 1998$, p.10). Strange, en collaboration avec Letizia Paoli, privilégie le cas de la mafia italienne pour illustrer cet argument dans The Retreat of the State. La mafia incarne pour elle le passage du crime organisé transnational, en tant qu'acteur non-étatique, de l'échelle locale à l'échelle mondiale (1996, p. 111). Elle observe que Cosa Nostra, dont les ressources financières augmentent grâce au marché lucratif des drogues illégales et aux réseaux de blanchiment de l'argent (1996, pp. 113-114 ; 1998 p. 128), est « le modèle d'un contregouvernement organisé » défiant l'Etat dans les différentes dimensions du pouvoir structurel (1996, p.110). Strange établit également des parallèles rapides entre des groupes de crime organisé italiens autres que Cosa Nostra et ceux d'autres pays. Elle note par exemple la façon dont la Mafia «s'apparente par certains traits à d'autres organisations illégales comme les triades chinoises, le cartel des drogues colombien, les groupes de yakusa japonais ou les milliers de mini-mafias récentes qui se sont formées dans l'ancienne Union soviétique » (1996, citation p. 93, pp. 111-112 ; 1998, p.10). Tout en reconnaissant que le crime organisé est un phénomène ancien, Stange affirme que l'émergence de réseaux mondiaux reliant les groupes criminels de différents pays constitue une nouveauté. Elle soutient que la prolifération de marchés lucratifs illégaux a centralisé les groupes criminels au niveau national et élargi le champ des interactions transnationales entre organisations criminelles. Il en résulte une forme de « diplomatie transnationale » entre "mafias nationales" fondée sur un «intérêt commun » à exploiter les marchés illégaux. Cette diplomatie a donné naissance à une prolifération d'«accords informels» qui dénotent selon Strange l'existence d'« une 'société internationale' anarchique de mafias comme il en existe une d'Etats » (1996, pp. 111-113). Strange relie la croissance du crime organisé transnational à l'émergence d'un marché mondial des drogues illégales et à des mesures gouvernementales qui rendent passibles de poursuites pénales «les échanges de biens ou de services » (1996, citation p. 114 ;1998, p.128). Elle observe la façon dont cette pénalisation a augmenté les risques, la rentabilité et les rentes de monopole pour les fournisseurs et, par là, encouragé la diversification des groupes du crime organisé (1996, pp. 114 ; 1998, p.10, p. 23). Elle énonce que les "opérateurs financiers ", surtout les banques, se trouvent de plus en plus en mesure de cacher l'origine et les connexions politiques de l'argent sale. Leurs actions sont facilitées par l'existence des paradis off-shore pour les fonds provenant de l'évasion fiscale, du détournement et des activités frauduleuses d'acteurs tant étatiques que non-étatiques (1996, citation pp. 117-119; 1998, pp. 123-125, pp. 134-135). Strange va plus loin en soutenant que les gouvernements ont négligé de prendre les mesures appropriées contre le blanchiment des profits générés par ces activités, mettant «en danger l'ensemble du système d'autorité étatique » (1996, pp. 117-119). Elle considère dans ce contexte les efforts sélectifs d'application répressive de la loi aux Etats-Unis et de la part d'organismes multilatéraux tels que le Groupe d'Action Financière Internationale (GAFI) comme des réponses en grande partie futiles 
aux exigences politiques nationales d'action (1998, pp. 124-126). Elle observe que ces demandes de contrôle entrent en conflit avec d'autres considérations politiques nationales et internationales qui ont dissuadé les gouvernements de faire obstacle aux changements continuels de pouvoir structurel dans le domaine de la finance (1998, pp. 130-135). L'expansion du crime organisé transnational constitue ainsi un des prix à payer pour cette inaction. Les ressources financières générées par la drogue et le blanchiment de l'argent ont selon elle modifié les relations entre les groupes de crime organisé transnational et l'Etat. En termes simples, "la richesse a donné du pouvoir » aux groupes criminels (1996, p. 115). Son analyse du cas de l'Italie lui donne l'occasion de noter que le pouvoir croissant de la mafia a changé les relations auparavant symbiotiques avec l'Etat, donnant naissance à des manifestations de violence et de répression gouvernementale. Strange extrapole à partir de ce cas pour avancer que la diffusion du pouvoir a contribué à user "la coexistence pacifique » et les nouveaux efforts d'accommodement entre les autorités étatiques et non-étatiques. Elle conclut du cas de l'Italie que « les problèmes ne commencent, en bref (comme dans les relations entre Etats), que lorsque les gouvernements d'Etats ayant jusqu'ici toléré la coexistence d'une autorité non-étatique rivale, perçoivent un important changement dans l'équilibre du pouvoir qui vient menacer leur propre survie» (1996, pp. 115-121, citation p. 120). Dans ses deux ouvrages, Strange se montre moins qu'optimiste sur les options possibles des autorités étatiques face à ces menaces, surtout du fait de la nature transnationale du crime organisé. Elle note, par exemple, que de nouveaux accommodements entre les autorités étatiques et le crime transnational organisé requerraient de relier entre elles « deux sociétés anarchiques ", une tâche « douteuse et improbable» (1996, p. 121). De façon analogue, le succès des tentatives transnationales de répression s'est vu limité par la réticence des gouvernements étatiques à céder de l'autorité aux institutions internationales (1996, pp. 119-121; 1998, pp. 132-136). En effet, bien que les Etats puissent recourir de façon croissante à des activités de réglementation, Strange affirme que la médiocre "qualité de cette autorité " est révélatrice de la diffusion du pouvoir de l'Etat au profit du crime transnational organisé (1996, p. xiv). Une étude du crime axée sur l'Etat Strange reproche aux spécialistes des relations internationales d'avoir omis de " proposer des théories explicatives pouvant s'adapter à l'émergence du crime organisé transnational en tant que menace majeure peut-être la menace majeure pour le système mondial à partir des années quatre-vingtdix » (1996, p. 121). Son appel en faveur d'une approche théoriquement informée de cette menace constitue une étape importante. Celle qu'elle propose n'en comporte pas moins un certain nombre de défauts critiques. Robert Gilpin (2000, p. 198) observe que ses ouvrages ultérieurs dénotent une évolution de sa "démarche intellectuelle » vers une approche non plus centrée sur l'Etat mais davantage "axée sur le marché ", mettant en avant les marchés et les acteurs transnationaux. Son analyse du crime organisé transnational porte cependant l'empreinte de son optique précédente. Tous les défauts qu'elle y relève ne l'empêchent pas moins de se fonder sur un certain nombre d'éléments clé de cette approche pour étudier les groupes criminels et les menaces qu'ils présentent. Elle définit de façon spécifique les groupes criminels organisés transnationaux comme des acteurs unitaires liés dans une société anarchique et engagés dans des transitions d'équilibre de pouvoir par rapport aux Etats. En conséquence, elle ne s'attache pas assez à explorer la nature des groupes criminels et elle surestime la menace qu'ils posent au pouvoir étatique. Les groupes criminels, acteurs unitaires. L'argument posant les Etats comme des acteurs unitaires renvoie 
habituellement aux œuvres de Kenneth Waltz (1979). Ce dernier privilégie les Etats dont il fait les acteurs primordiaux de la politique internationale. Il les définit comme «semblables à des unités» dans les fonctions qu'ils accomplissent ou tentent d'accomplir par définition et soutient que les différences entre eux résident principalement dans les capacités qu'ils peuvent consacrer à ces tâches. Cherchant à développer une analyse systémique de la politique internationale, Waltz s'est peu penché sur les variations des caractéristiques internes de ces unités pour souligner au contraire la répartition du pouvoir entre elles (1979, pp. 96-97, pp. 130-131). Il est clair que Strange a voulu dépasser cette vision des Etats en acteurs unitaires et a rejeté une approche systémique stato-centrique définissant le pouvoir en termes de répartition des capacités. Or, suivant Waltz en cela, elle étudie les groupes criminels dans ses deux ouvrages en les posant comme des unités semblables du point de vue du fonctionnement et en accordant peu d'attention aux variations de leurs caractéristiques internes. Elle affirme que les groupes criminels cherchent des rentes de monopole issues d'activités illégales, en recourant à la violence lorsqu'il est nécessaire de protéger ces rentes. Elle professe qu'ils se sont de plus en plus centralisés au niveau national, profitant de et contribuant à l'évolution des marchés de la drogue et des opportunités de blanchiment à l'échelon local, régional et national. Elle énonce également qu'ils étendent leurs réseaux de trafic de drogue à la fourniture d'autres produits et services illégaux. Strange considère en fait que ces similarités contribuent à expliquer pourquoi les groupes de crime organisé transnational défient le pouvoir étatique (1996, pp.111-114;1998, pp. 128-129). Les économistes étudiant la mafia " comme une entreprise commerciale comme une autre " sous-estiment l'impact social et politique plus large du crime organisé (1998, p. 10, p. 21 note 4). Elle ne s'en fonde pas moins implicitement sur les arguments de ces mêmes économistes pour édicter sa thèse de base selon laquelle les groupes criminels sont analogues du point de vue fonctionnel, cherchant tous à obtenir des rentes de monopoles dans des activités illégales (voir par exemple Fiorintini et Peltzman 1995, pp. 7-12 ; Strange 1996, p.114). Or, ces aperçus sur la poursuite du profit mis à part, les publications sociologiques et économiques révèlent que les groupes de crime organisé transnational ne sont pas tous semblables et ces différences comptent. Celles-ci peuvent se regrouper dans deux catégories, la fonction et l'organisation. Les groupes criminels diffèrent dans les fonctions qu'ils accomplissent. La plupart cherchent, certes, des rentes de monopole, mais certains d'entre eux agissent comme des pourvoyeurs à divers titres de protection privée alors que d'autres préfèrent assumer des rôles plus limités dans le trafic de biens et de services choisis (par exemple Schelling 1976; Gambetta 1993; Ruggiero 1996; Williams 1998). Dans bien des cas, les réseaux criminels peuvent s'étendre pour faciliter les mouvements d'un vaste ensemble de biens et de services (notamment Clawson et Lee 1996, pp. 88-89). Or, et pour toute une série de raisons, les groupes criminels refusent souvent cette option. Les groupes criminels diffèrent également dans leurs caractéristiques internes. La plus importante d'entre elles est bien évidemment le degré d'organisation réelle du crime organisé. Cette question reste très contestée parmi les spécialistes des sciences sociales. Strange analyse brièvement les systèmes de liens entre les groupes criminels, mais en omettant de participer au débat ancien sur l'étendue et la nature de l'organisation au sein de ces groupes (voir notamment Cressey 1969 ; Albini 1971 ; Gambetta 1993, pp. 100-126 ; Ruggiero 1996, pp. 133-134 ; Williams 1998). Elle surestime par conséquent le degré de concentration de ces groupes à l'échelon national. Elle observe des schémas analogues de centralisation et de 
coordination au sein des groupes criminels et les uns avec les autres, citant de façon explicite les cas du Japon et de l'Italie. Elle interprète mal celui du Japon en ceci qu'elle ne saisit pas les variations importantes des liens relationnels informels et formels parmi les principaux groupes de yakusa et entre ceux-ci (Friman 1999 ; sur l'Italie, voir Gambetta 1993 ; Clawson et Lee 1996, pp. 62-86). Strange exagère en fait l'importance du défi posé aux Etats par les groupes de crime organisé en négligeant leurs caractéristiques internes. L'autre société anarchique. Les spécialistes contestent également les schémas de relations organisées entre les groupes criminels (par exemple Naylor 1995 ; Clawson et Lee 1996; Shelley 1999). Strange rend brièvement compte de ce débat dans The Retreat of the State, notant la thèse de la journaliste Claire Sterling sur l'existence d'une pax mafiosa qui lierait formellement les organisations de crime transnational. Mais notre auteur passe finalement à côté de la question. Elle conclut, en un saut logique incorrect, à la primauté de l'existence d'« une sorte de 'société internationale' anarchique de mafias à l'image de celle des Etats », quelle que soit la nature des liens entre les groupes de crime organisé (1996, pp. 112-113). L'argument stato-centrique posant l'Etat comme membre d'une société anarchique remonte aux travaux de Hedley Bull (1977). Bull a cherché à remettre en cause la thèse de chercheurs réalistes selon laquelle l'absence d'autorité supranationale - la définition réaliste de l'anarchie adoptée par Waltz et par d'autres - empêche l'existence d'un ordre international. Il soutient qu'un « élément de société internationale » existe dans les relations entre Etats. Bull définit cette société comme le développement de « règles et d'institutions » fondées non seulement sur un simple aspect pratique mais aussi sur des « impératifs de moralité et de légalité » (1977, pp. 26-27, p. 42). Ce contraste entre systèmes de liens procédant de la commodité et l'émergence de normes et de principes plus importants se trouve au cœur du débat sur la nature du crime organisé transnational. Si l'aspect pratique constitue la caractéristique fondatrice des relations entre les groupes criminels, l'hypothèse de Strange de l'existence d'une société anarchique comparable à celle que forment les Etats a peu de raisons d'être. Si des règles et des institutions formelles plus importantes existent, la représentation qu'elle fait de la société anarchique présente une certaine plausibilité. Il est cependant clair que sa thèse privant le débat sur la nature d'une pax mafiosa de toute importance et posant les sociétés anarchiques d'Etats et les groupes criminels comme comparables "dans tous les cas ", est défectueuse et n'offre que des aperçus limités sur la nature de ces derniers (1996, p.113). Equilibre et transition. Selon notre auteur, les activités illégales des groupes de crime transnational rendent conflictuelle par essence l'intersection des sociétés anarchiques de mafias et d'Etats (1996, pp. 119-121). Bien que les Etats puissent tenter d'éradiquer les activités illégales, elle constate que leurs gouvernements ont, parfois, cherché des accommodements implicites ou explicites avec les mafias. Les groupes criminels supplantent toujours l'autorité étatique dans ce contexte mais les conditions de ces accommodements jouent en sens inverse. Strange soutient que la possibilité de maintenir cette "coexistence pacifique symbiotique " dépend d'un équilibre de pouvoir entre l'autorité étatique et non-étatique (1996 p. 119). Or la coexistence peut s'éroder pour faire place à la violence et à la suppression de l'activité illégale si cet équilibre est perturbé. Extrapolant à partir du cas de l'Italie, Strange affirme que le marché a bouleversé l'équilibre du pouvoir en « confér[ant] une nouvelle richesse (et un nouveau pouvoir) au crime organisé » et en faisant prendre conscience aux gouvernements des Etats territoriaux que leur survie est en jeu. Elle observe que, "comme dans les relations entre les Etats", les changements dans 
l'équilibre du pouvoir entre autorités étatiques et non-étatiques sont automatiquement porteurs de conflit (1996, pp. 118-121). Cette comparaison implicite avec des arguments stato-centriques sur l'équilibre du pouvoir pose cependant plus de questions qu'elle n'en résout. La relation entre équilibre du pouvoir et conflit, notamment, reste une question controversée pour les spécialistes des relations internationales. La littérature à ce sujet abonde en hypothèses contraires selon lesquelles les équilibres de pouvoir, un rapprochement ou un éloignement de ces équilibres, débouchent sur la paix ou sur la guerre (par exemple les analyses de Tammen et al. 2000). La difficulté réside en partie dans la définition du concept, car on ne sait quelles sont les répartitions du pouvoir qu'on peut précisément qualifier d'équilibre (voir par exemple les comptes rendus de Viotti et Kauppi 1993, p. 64 ; Burchill et Linkater 1996, pp. 33-34, pp. 39-41). De façon analogue, Strange soutient que la tendance à un déséquilibre de pouvoir mène au conflit sans toutefois parvenir à mesurer de façon appropriée l'évolution du pouvoir que ce mouvement implique. Elle évite ce problème de façon implicite en suggérant que l'évolution du pouvoir réelle, imputable au marché est moins importante que la menace correspondante perçue par les gouvernements. Cette distinction, malgré son importance, soulève des questions sur la nature des conditions influant sur la perception de la menace (voir notamment Jervis 1978). Hormis une analyse rapide du cas de l'Italie, Strange ne propose pas d'étude fouillée des conditions dans lesquelles les gouvernements sont les plus susceptibles de percevoir comme une menace pour leur propre survie des changements dans le pouvoir des groupes criminels imputables au marché. Les relations d'équilibre de pouvoir, et principalement la perception des menaces pour la sécurité, sont également interactives. Or Strange n'analyse pas la manière dont les groupes de crime organisé perçoivent l'évolution du marché comme un facteur de changement de leur pouvoir par rapport aux Etats. Cette lacune affaiblit sa conclusion sur les perspectives d'accommodement. Les théoriciens de la transition du pouvoir observent que le potentiel d'accommodement dépend de la perception des éléments puissants autant que de ceux dont le pouvoir augmente (par exemple Organski et Kugler 1980 ; Tammen et al. 2000). Strange s'attache seulement à considérer une moitié de cette relation. La conviction de l'existence de la menace La relation entre la perception et la réalité de la remise en cause du pouvoir étatique joue un rôle central dans l'analyse plus large de Strange. Elle souligne que la mesure dans laquelle l'autorité non-étatique défie celle de l'Etat «n'est pas, et ne peut pas être, déterminée objectivement " (1996, p. 92), mais « dépend totalement de la perception de l'Etat, soit qu'il considère l'autorité non-étatique comme un allié..., soit qu'il voit en elle un ennemi, un rival en termes de légitimité et de pouvoir» (1996, p. 92). Et cependant, le problème tel que le voit Strange est que « les chefs de gouvernement peuvent être les derniers à reconnaître qu'eux-mêmes et leurs ministres ont perdu l'autorité qu'ils détenaient sur les sociétés et les économies nationales » $(1996$, p. 3). Elle affirme que le crime organisé transnational constitue une menace réelle qui doit être perçue par les gouvernements et les chercheurs du fait de la nature même des défis qu'il pose à l'autorité étatique. Or elle considère en même temps l'Etat comme incapable d'inverser la diffusion du pouvoir vers le crime organisé. Elle fustige les grands noms de la recherche pour leur absence de reconnaissance de la "réalité du pouvoir du marché » et de la «diffusion de l'autorité réelle sur l'économie et la société » (1996, p.121). Elle observe précisément que le crime organisé vient clairement menacer au moins deux «responsabilités" essentielles "dévolues à l'Etat», soit le pouvoir de l'Etat de percevoir des recettes et son monopole sur l'emploi de la violence à l'intérieur de ses 
frontières territoriales (1996, p. 77, p. 82). L'hypothèse de base selon laquelle le crime transnational constitue une menace sérieuse a des mérites considérables (par exemple Friman et Andreas 1999), mais la façon dont Strange y adhère risque de diminuer sa faculté d'analyse critique. Elle aborde en effet le discours de plus en plus fréquent des responsables officiels sur le crime organisé transnational comme une réponse extrêmement tardive, quoique futile, à une réalité menaçante. Elle accorde par conséquent peu d'attention à l'analyse critique de ce discours. Réalités « incontestables ». Strange s'appuie sur les statistiques officielles pour illustrer la façon dont l'évolution du marché a profité au pouvoir du crime organisé transnational. Malgré la croissance d'autres activités, le trafic de drogue et le blanchiment de l'argent jouent un rôle central dans son analyse de cette expansion. Elle affirme que si le débat sur l'existence d'une pax mafiosa reste peu concluant, «la flambée des statistiques criminelles et la facilité du passage des biens et des personnes par delà les frontières nationales [laquelle favorise la mondialisation des activités du crime organisé] sont des faits incontestables » (1996, pp. 112-113). Le progrès technique et la déréglementation étatique des flux transnationaux ont ouvert des opportunités en ce qui concerne l'activité criminelle et les défis portés au pouvoir étatique (voir notamment Williams 1998 ; Findlay 1999 ; Friman et Andreas 1999). Les statistiques concernant le trafic de la drogue et le blanchiment de l'argent se trouvent par ailleurs en augmentation. Les chiffres de la criminalité sont cependant loin d'être incontestables. Strange affirme que les marchés de la drogue et le blanchiment de l'argent ont connu un essor depuis les années soixante, mais elle adopte largement et sans le remettre en question le discours des organismes de répression sur l'importance de cet essor. Elle reprend par exemple les données des Nations Unies sur les saisies de drogue sans prendre en compte l'aspect problématique des pratiques de recensement sur lesquelles ces chiffres se fondent. Elle suppose que l'existence d'un taux constant de saisies de drogue dans le temps va dans le sens de l'argument d'un essor du marché de la drogue mais elle omet de reconnaître les variations importantes des efforts d'interdiction entre Etats et sur une longue période (Strange 1996, pp. 113-114). Les rapports officiels de saisie de drogue aux EtatsUnis, en Europe et au Japon, font état d'une interdiction allant de 4 à $30 \%$ et plus, de l'ensemble du commerce de la drogue. Les organismes concurrents de répression, à la recherche d'une reconnaissance de leur action, se sont également engagés dans un double compte des mêmes quantités de drogue saisie (par exemple Clawson et Lee 1996, pp. 4-25; Friman 1996; Stares 1996, citation p. 11). Strange affirme que même les estimations conservatrices du GAFI et du Parlement européen sur le blanchiment de l'argent lié à la drogue « confirment » sa thèse et son argumentation plus large (Strange 1996, p.114), tout en omettant d'en reconnaître, là encore, la nature problématique. Les estimations qui concernent le blanchiment de l'argent se fondent souvent sur les données de saisie peu fiables citées plus haut multipliées par des estimations des prix moyens pratiqués lors des ventes de rue. D'autres chiffres résultent de formules contestables fixant approximativement la marge bénéficiaire revenant aux groupes criminels aux divers stades du commerce de la drogue, ou multipliant les prix de la vente de rue par les schémas de consommation approximatifs des populations dépendantes estimées, ou encore calculant des pourcentages approximatifs d'après le poste des décalages statistiques des chiffres de la balance nationale des paiements (par exemple Smith 1992; Clawson et Lee 1996, pp.4-25; Reuter 1996). Les chiffres sur le blanchiment de l'argent, notamment la somme de 100 milliards de dollars générés par le commerce de la drogue et 150 de plus par d'autres activités de contrebande dans le 
milieu des années quatre-vingt-dix, représentent pour Strange des indicateurs du pouvoir du crime organisé à se procurer des recettes (1996, p.114;1998, p.124). Or elle ne se soucie pas, à l'image d'un certain nombre de rapports officiels, de placer ces chiffres de façon appropriée dans un contexte comparatif. Elle ne donne aucune idée de la façon dont ce flux de recettes se répartit par groupe criminel sauf à faire de brèves références à l'accroissement de richesse de ces groupes en Italie et en Colombie. Ses arguments impliquent au contraire que les groupes de crime organisé ont partagé cette manne de façon égale et présentent chacun une menace semblable pour le pouvoir étatique. On ne peut que s'interroger quant à cette affirmation, étant donné l'importante variation des caractéristiques des groupes criminels que nous avons notée plus haut. Strange n'étudie pas non plus de façon appropriée la dynamique en matière de recettes du crime organisé dans le contexte d'autres flux financiers transnationaux. Elle reconnaît dans ses deux ouvrages que d'autres types d'activités financières illicites que le blanchiment de l'argent, comme l'évasion fiscale, le détournement de fonds et la fraude, représentent des menaces plus sérieuses pour le pouvoir étatique. Mad Money consacre néanmoins un chapitre entier à l'aspect illégal de la finance sans offrir un seul chiffre sur ces activités (1996, p. 117 ; 1998, pp. 123-136). Dans des chapitres séparés de The Retreat of the State, elle révèle que le danger que représentent les recettes des groupes de crime organisé n'est pas nécessairement plus important que ceux provenant d'autres acteurs non-étatiques. Les groupes criminels se situent entre les entreprises générant les 530 milliards de dollars annuels du marché des télécommunications et les 30 milliards annuels d'honoraires des six grandes entreprises de comptabilité (1996, p. 104, p. 135). Fait plus important, les chiffres de Strange sur le blanchiment de l'argent restent encore faibles en comparaison des recettes de l'Etat. L'estimation totale de 250 milliards de dollars blanchis au milieu des années quatre-vingt-dix apparait plutôt mince par rapport aux 6000 milliards de dollars du produit intérieur brut des EtatsUnis, sans parler de celui des 50 Etats les plus importants de l'économie mondiale au début de la décennie (Lairson et Skidmore 1997, pp. 112-115). La construction du crime. Strange s'intéresse peu aux causes du discours gouvernemental sur la menace majeure que représente le crime organisé transnational pour le pouvoir étatique. Comme nous l'avons noté, elle pose comme hypothèse de base l'existence d'un défi réel, surtout face aux prérogatives du pouvoir étatique en matière de violence et de finances, que les gouvernements se sont montrés par trop lents à admettre. Son analyse soulève néanmoins des questions quant à l'importance de ces menaces par rapport à d'autres et révèle son incapacité à saisir les raisons pour lesquelles certains problèmes sociaux viennent dominer le discours gouvernemental. Dans les deux ouvrages, elle met l'accent sur la question de l'autorité de l'Etat sur les recettes plutôt que sur la violence. Son analyse en elle-même de la violence exercée par les groupes criminels est rapide. Elle est cependant moins limitée par sa brièveté que par son ancrage sur les Etats relativement faibles de l'Italie, de la Colombie et de l'ancienne Union soviétique. Il est clair que ces Etats ont subi la violence du crime organisé. Strange surestime cependant le cas de la Colombie en prenant en compte la violence extrême du cartel de Medellin de 1984 à 1990 et en omettant son effondrement en 1993 du fait d'une campagne de répression de la part de l'Etat (1996, p. 117). L'étude de l'expérience d'Etats plus forts constituerait un test plus solide du défi posé par le crime organisé. Strange note en passant que la capacité des groupes criminels à accéder aux marchés mondiaux des armes étend la menace à ces pays (1996, pp. 81-82, pp. 115-117). Mais les seuls cas qu'elle cite sont la secte d'Aum Shinrikyo au Japon et, dans une référence générale, les 
groupes terroristes (1996, p. 82). Il est clair que les groupes criminels peuvent recourir à la violence et aux menaces de violence et qu'ils le font occasionnellement. La violence leur offre un moyen de maintenir l'ordre dans leurs rangs et de consolider leur part de marché face à leurs concurrents (Strange 1996, p. 110). Or l'expérience des organisations de Medellin et de Cali en Colombie révèle que les groupe criminels ne sont pas tous également violents (par exemple Lee 1989). Fait plus important, ceux-ci tendent à considérer la violence comme " peu propice aux affaires ", contrairement au culte d'Armageddon et aux organisations terroristes, sans parler d'autres acteurs nonétatiques comme les forces paramilitaires de droite et les guérillas de gauche, et cherchent plutôt des accommodements et des arrangements organisationnels (par exemple Schelling 1976 ; Ruggiero 1996). Strange n'explique cependant pas pourquoi les groupes de crime organisé transnational sont désignés par les gouvernements comme la principale source de menace pour l'autorité étatique, plutôt que d'autres acteurs non-étatiques dotés d'un potentiel de violence plus important. Notre auteur cherche à proposer une thèse plus solide lorsqu'elle aborde la menace posée à l'autorité étatique en matière de recettes. Elle affirme que les groupes de crime organisé transnational s'orientent de façon croissante vers le système financier mondial (1998, p. 123), tout en admettant, malgré cette tendance, que le blanchiment d'argent par le crime organisé ne constitue qu'une petite partie des activités financières internationales illégales. L'évasion fiscale, la fraude et le détournement de fonds restent les domaines de prédilection d'un grand nombre d'acteurs étatiques comme non-étatiques. Strange traite effectivement la question de savoir pourquoi les gouvernements ont donné la première place à la menace parmi d'autres du blanchiment de l'argent. Dans Mad Money par exemple, elle soutient que c'est la pression, sur la classe politique, de l'opinion publique et des médias en faveur de mesures contre le commerce de la drogue qui explique la mise en priorité de la part du gouvernement américain de cette activité plutôt que d'autres, tout aussi illégales, dans le domaine financier (1998, p. 126). A l'opposé, son analyse de l'évasion fiscale ne fait pas état de ces pressions. Elle y soutient plutôt que l'explication de l'absence relative de mesures contre les paradis fiscaux réside dans les pressions politiques d'anciennes colonies, des considérations de développement de pays étrangers et des "accidents historiques » (1998, pp.132-133). Or Strange se trompe dans ces deux cas en pensant les gouvernements comme des acteurs unitaires qui ne font que réagir à des considérations de politique nationale ou internationale (par ex. voir Helleiner 1999). L'hypothèse de base que fait notre auteur de l'existence de menaces réelles et de leur reconnaissance progressive par les gouvernements limite également sa capacité à expliquer la mise en priorité des menaces représentées par le blanchiment de l'argent. Elle blâme dans The Retreat of the State les gouvernements pour leur nonreconnaissance de ce phénomène et la non-pénalisation pleine et entière de celui-ci (1996, pp. 118-119). Deux ans plus tard, dans Mad Money, elle se montre plus cynique et dépeint les efforts de contrôle de ce phénomène comme une "pantomime " politique des responsables gouvernementaux conscients de la futilité de leurs efforts éventuels. La menace réelle en la matière semble résider pour elle dans l'insolubilité du problème. Elle affirme que les Etats peuvent fermer les paradis fiscaux et résoudre le problème de l'évasion fiscale s'ils en ont la volonté (1998, pp.188-189). Les forces du marché au contraire, l'intrication de la communauté bancaire, et la réticence des Etats à abandonner leur autorité souveraine sur l'application de la loi, placent le contrôle du blanchiment de l'argent hors de toute atteinte (1998, pp. 129-132). Elle ne se prononce 
pas sur le paradoxe selon lequel la volonté politique n'a pas de prise sur les questions auxquelles les gouvernements donnent priorité (le blanchiment de l'argent) et s'avère vitale dans celles traitées comme non prioritaires (les paradis fiscaux). Strange reconnaît que le crime reflète des contextes sociaux et culturels et que les paramètres définissant la criminalité pour un gouvernement peuvent changer au fil du temps (1996, p. 114, pp. 117-119; 1998, pp. 123-24). Mais, contrairement aux sociologues et aux criminologues, elle n'étudie pas l'interaction des acteurs étatiques et non-étatiques dans la construction sociale de la menace représentée par le crime. Les approches constructivistes analysent la façon dont un problème social comme la menace posée par le crime devient "construite, c'est-à-dire expliquée en termes de causes et de remèdes" par les hommes politiques, les responsables répressifs, les médias, les spécialistes universitaires et le public (par exemple Sasson 1995, p. 2 ; Welch, Fenwick, Roberts 1998, pp. 87-88; Ferrell et Websdale 1999, p. 10). Comme l'observe Joel Best (1998, pp. 221-222), les menaces sociales sont moins déterminées par des «faits objectifs » que par le " processus de déclaration » des acteurs étatiques et sociaux qui ont un "intérêt personnel à mettre une question en évidence ou à promouvoir une image particulière d'un problème ». Les efforts des personnes à l'origine de ces déclarations pour attirer l'attention sur des problèmes particuliers peuvent influencer la perception d'autres acteurs étatiques et sociaux. Ils peuvent dans certains cas aller jusqu'à « provoquer » des paniques morales lorsque les problèmes sociaux virent aux croisades morales contre des groupes ou des activités définis comme menaçant la " stabilité et le bien-être " mêmes " de la société » (par exemple Goode et Ben-Yehuda 1994, pp. 161-163; Potter et Kappeler 1998, p. 7 ; Thompson 1998, p. 7, p. 19 ; Findlay 1999, p. 53). Strange fait en effet partie de ces nombreux auteurs de déclarations faisant de la construction sociale du crime organisé transnational la principale menace envers le pouvoir étatique. Cette démarche reflète son hypothèse de base sur la réalité de cette menace mais la place trop près de ce processus pour pouvoir analyser de façon critique le discours gouvernemental sur «la nature de la menace... et la [ou les] riposte[s] au phénomène " (Naylor 1995, p. 50). Le meilleur exemple en est peut-être que Strange omet de reconnaître que le discours actuel sur le crime organisé transnational est tenu dans un monde de l'après-guerre froide dépourvu de toute "menace sur la sécurité nationale » comparable à celle qu'exerçait l'ancienne Union soviétique (Naylor 1995, p. 53). Dans ce contexte, les organismes étatiques de sécurité, y compris ceux de maintien de l'ordre et l'armée, ont intérêt à rendre prioritaires de nouvelles menaces comme celle-ci. Cette mise en priorité constitue pour eux un moyen d'obtenir des ressources, le soutien public et une extension de leurs compétences. Il est nécessaire de mener une recherche systématique pour déterminer le degré de construction sociale du crime organisé transnational, identifier les auteurs de déclarations et étudier l'impact de ce processus sur le pouvoir étatique. Mark Findlay (1999, pp. 48-49, p. 51, p. 102) observe que la mise en priorité récente du lien entre la menace posée par la drogue et le crime organisé constitue un exemple clair du caractère sélectif de la "politique de criminalisation ». R.T. Naylor constate que « le crime dans la société moderne » est une « menace sociale » grave (Naylor 1995, p. 50). Mais il souligne que les arguments faisant du crime organisé transnational la source du problème et préconisant comme solution une campagne nouvellement centrée sur celui-ci par les acteurs de la sécurité étatique, exigent davantage d'"analyse critique " qu'il n'en a été fait actuellement. Susan Strange a contribué à légitimer le travail de spécialistes visant à élargir le champ des relations internationales au-delà de ses paramètres orthodoxes. En soutenant que des 
acteurs étatiques et non-étatiques se disputent le pouvoir dans le contexte des relations transnationales, elle a remis en question l'optique centrée sur l'Etat de la discipline. L'élargissement de cette approche au crime organisé transnational dans ses derniers ouvrages ouvre la voie à la prise en compte dans cette discipline d'une question importante et non encore suffisamment explorée, notamment dans l'étude de l'économie politique internationale. Mais le chemin qu'elle nous convie à emprunter est semé d'embûches. Elle se fonde sur les concepts centrés sur l'Etat de la discipline qu'elle a cherchée à remettre en question, négligeant certains aspects de la nature des groupes criminels et surestimant la menace qu'ils représentent pour le pouvoir étatique. Procédant également des perceptions étatiques qui confirment son hypothèse de base sur la menace posée par le crime organisé transnational, son approche n'analyse guère de façon critique le discours des responsables gouvernementaux et la construction sociale plus large des menaces que constitue le crime. Les voies novatrices, même semées d'embûches, invitent à l'exploration. Strange observe que « les gangs criminels organisés... existent depuis longtemps » (1996, p.110). Son travail de recherche met au défi les spécialistes des relations internationales de leur porter enfin attention.

2 Bibliographie Albini J. L., The American Mafia: Genesis of a Legend, New York, Appleton-Century-Crofts, 1971. Best J., 'Road Warriors' on 'Hair-Trigger Highways', Cultural Resources and the Media's, 1998. "Construction of the Freeway Shootings Problem ", in Potter G.W. et Kappeler V. E. (dir.), Constructing Crime : Perspectives on Making News and Social Problems, Prospect Heights, I.L., Waveland Press, 1987, pp. 221-40. Bull H., The Anarchical Society : A Study of Order in World Politics, New York, Columbia, University Press, 1977. Burchill S. et Linklater A., Theories of International Relations, New York, St.Martin's Press, 1996. Clawson P. L. et Lee R., The Andean Cocaine Industry, New York, St. Martin's Press, 1996. Cressey D. R., Theft of the Nation : The Structure and Operations of Organized Crime in America, New York, Harper and Row, 1969. Ferrell J. et Websdale N., « Materials for Making Trouble ", in Ferrell J., et Websdale N. (dir.), Making Trouble: Cultural Constructions of Crime, Deviance, and Control, New York, Aldine de Gruyter, 1999, pp. 3-24. Findlay M., The Globalization of Crime : Understanding Transnational Relationships in Context, Cambridge, Cambridge University Press, 1999. Fiorintini G. et Peltzman S., «Introduction », in Fiorentini G. et Peltzman S. (dir.), The Economics of Organized Crime, Cambridge, Cambridge University Press, 1995, pp.1-30. Friman H. R., NarcoDiplomacy : Exporting the U.S. War on Drugs, Ithaca, Cornell University Press, 1996. Friman H. R., "Obstructing Markets, Organized Crime Networks and Drug Control in Japan ", in Friman H.Richard et Andreas Peter (dir.), The Illicit Global Economy and State Power, Boulder, Rowman and Littlefield, 1999, pp.173-197. Friman H. R. et Andreas P. (dir.), The Illicit Global Economy and State Power, Boulder, Rowman and Littlefield, 1999. Gambetta D., The Sicilian Mafia : The Business of Private Protection, Cambridge, Harvard University Press, 1993. Gilpin R., "The Retreat of the State ", in Thomas C. Lawton, Rosenau J. et Verdun A. C. (dir.), Strange Power: Shaping the Parameters of International Relations and International Political Economy, Aldershot, Ashgate, 2000, pp. 197-213. Goode E. et BenYehuda N., "Moral Panics : Culture, Politics, and Social Construction », Annual Review of Sociology, 20, 1994, pp. 149-171. Helleiner E., «State Power and the Regulation of Illicit Activity in Global Finance », in Friman H. R. et Andreas P., (dir.), The Illicit Global Economy and State Power, Boulder, Rowman and Littlefield, 1999, pp. 53-90. Jervis R., "Cooperation under the Security Dilemma", World Politics 30 (January), 1978, pp. 
167-214. Lairson Th. D. et Skidmore D., International Political Economy : the Struggle for Power and Wealth, New York, Harcourt Brace, 1997. Lawton Th. C., Rosenau J. et Verdun A. C., (dir.), Strange Power : Shaping the Parameters of International Relations and International Political Economy, Aldershot, Ashgate, 2000. Lee R., The White Labyrinth: Cocaine and Political Power, New Brunswick, N.J., Transaction Publishers, 1989. Naylor R.T, «From Cold War to Crime War: The Search for a New 'National Security' Threat », Transnational Organized Crime, 1, 4 (winter), 1995, pp. 37-56. Organski A.F.K. et Kugler J., The War Ledger, Chicago, University of Chicago Press, 1980. Potter G. W. et Kappeler V. E., «Introduction », in Potter Gary W. and Kappeler Victor E. (dir.), Constructing Crime : Perspectives on Making News and Social Problems, Prospect Heights, I.L., Waveland Press, 1998, pp.1-24. Reuter P., « The Mismeasurement of Illegal Drug Markets ", in Pozo S. (dir.), Exploring the Underground Economy, Kalamazoo, M.I., W.E. UpJohn Institute, 1996, pp.63-80. Sasson Th., Crime Talk: How Citizens Construct a Social Problem, New York, Aldine de Gruyter, 1995. Schelling Th. C., "Economic Analysis and Organized Crime», in Task Force Report, The President's Commission on Law Enforcement and the Administration of Justice, Washington, D.C., GPO, 1976, pp.114-126. Shelley L. I., «Transnational Organized Crime: The New Authoritarianism ", in Friman H. Richard and Andreas Peter (dir.), The Illicit Global Economy and State Power, Boulder, Rowman and Littlefield, 1999, pp. 25-52. Stares P., Global Habit: The Drug Problem in a Borderless World. Washington, D.C., The Brookings Institution, 1996. Ruggiero V., Organized and Corporate Crime in Europe, Aldershot, Dartmouth Publishing, 1996. Smith P. H., « The Political Economy of Drugs : Conceptual Issues and Policy Options », in Smith Peter H. (dir.), Drug Policy in the Americas, Boulder, Westview Press, 1992, pp. 1-21. Strange S., States and Markets, London and New York, Pinter, 1988. Strange S., The Retreat of the State : The Diffusion of Power in the World Economy, Cambridge, Cambridge University Press, 1996. Strange S., Mad Money, Manchester, Manchester University Press, 1998. Tammen R. L., et al., Power Transitions : Strategies for the 21st Century, New York and London, Chatham House, 2000. Thompson K., Moral Panics, London, Routledge, 1998. Viotti P. R. et Kauppi M. V., International Relations Theory: Realism, Pluralism,Globalism, New York, MacMillan, 1993. Waltz K., Theory of International Politics, Reading, M.A., AddisonWesley, 1979. Welch M., Fenwick M. et Roberts M., « State managers, Intellectuals and the Media ", in Potter Gary W. and Kappeler Victor E. (dir.), Constructing Crime : Perspectives on Making News and Social Problems, Prospect Heights, I.L., Waveland Press, 1998, pp. 87-110. Williams Phil., «Transnational Criminal Organization and International Security », in Klaare M. et Chandrani Y. (dir.), World Security : Challenges for a New Century, New York, St. Martin's Press, 1998, pp. 249-272.

\section{NOTES}

1. Texte traduit de l'anglais par Marie-Pierre Emery 
INDEX

Mots-clés : économie politique, Etat-nation, mafias et crime organisé, Relations Internationales, réseaux transnationaux 